Classification

Physics Abstracts

71.20

\title{
The electronic and magnetic properties of $\mathrm{Fe}_{3} \mathrm{~N}$
}

\author{
S. Matar $\left({ }^{1,3, *}\right)$, B. Siberchicot $\left({ }^{2}\right)$, M. Pénicaud $\left({ }^{2}\right)$ and G. Demazeau $\left({ }^{3}\right)$ \\ (1) Technische Hochschule Darmstadt, Fachbereich Physik, FB5, D-6100 Darmstadt, Germany \\ (2) C.E.A. Centre d'Etudes de Limeil-Valenton, 94195 Villeneuve St-Georges Cedex, France \\ ( ${ }^{3}$ ) L.C.S.-C.N.R.S., Université Bordeaux 1, 33405 Talence Cedex, France
}

(Received 7 February 1992, accepted in final form 4 May 1992)

\begin{abstract}
Résumé. - Les propriétés électroniques et magnétiques du nitrure ferromagnétique $\mathrm{Fe}_{3} \mathrm{~N}$ ont été étudiées par la méthode de l'onde sphérique augmentée ASW. Les calculs ont été effectués dans deux structures cristallines différentes: les structures hexagonale (expérimentale) et $\mathrm{Cu}_{3} \mathrm{Au}$ (hypothétique) afin d'établir des corrélations entre la structure magnétique de $\mathrm{Fe}_{3} \mathrm{~N}$ et celle de ses homologues $\mathrm{Fe}_{3} \mathrm{X}$ (X= non-métal). Les résultats sont discutés en connection avec des résultats expérimentaux (mesures d'aimantation, résonance Mössbauer). Indépendamment de la structure, des densités d'états dues à la liaison fer-azote sont introduites au niveau de Fermi et devraient jouer un rôle dans le magnétisme du nitrure.
\end{abstract}

\begin{abstract}
The electronic and magnetic properties of the ferromagnetic nitride $\mathrm{Fe}_{3} \mathrm{~N}$ were investigated by use of the ASW method. The calculations were done in two different crystal structures : the experimental-hexagonal one and a hypothetical $\mathrm{Cu}_{3} \mathrm{Au}$-type one- in order to correlate the magnetic structure of $\mathrm{Fe}_{3} \mathrm{~N}$ with that of its homologues $\mathrm{Fe}_{3} \mathrm{X}(\mathrm{X}=$ non-metal). Results were assessed in connection with existing experimental data (magnetic measurements, Mössbauer resonance). Independently of the structure, densities of states due to the iron-nitrogen bond are introduced at Fermi level and should play a role in the magnetism of the nitride.
\end{abstract}

\section{Introduction.}

The iron-nitrogen system has been largely investigated since the beginning of the century [ 1 , 2a]. Within this system, $\varepsilon$-iron nitride is a hexagonal phase extending over a wide, temperature dependent domain of existence [2b] (Tab. I). This phase is generally represented by the $\mathrm{Fe}_{3} \mathrm{~N}$ stoichiometric composition where nitrogen occupies alternate octahedral interstices in an ordered manner (Fig. 1). This is one reason why this ferromagnetic nitride has the highest Curie point ( $T_{\mathrm{C}} \cong 567 \mathrm{~K}$ [3]) within the $\varepsilon$-phase [4]. The magnetic properties of nitrogen-excess $\varepsilon$-iron nitride were investigated by neutron diffraction as well as by bulk magnetic measurements [5] (Tab. I, row 6). A low magnetic moment per Fe of $1.33 \mu_{B}$ was measured and confirmed by neutron diffraction results $\left(1.5 \mu_{\mathrm{B}}\right)$. Discussing the character of

(*) Permanent address : L.C.S.-C.N.R.S., Université Bordeaux 1, 33405 Talence Cedex, France. 
the Fe.. $\mathrm{N}$ bond, it was argued [5] that nitrogen does not act as an electron acceptor in the material as it would be expected for an ionic picture of a nitride. Elsewhere [6] Mössbauer spectroscopy and magnetic measurements of nitrogen deficient $\varepsilon$-iron nitride were interpreted on the assumption of nitrogen acting as an electron donor to Fe. That would explain the regular increase of the isomer shift and the decrease of internal field with increasing amount of $\mathrm{N}$ within the $\varepsilon$-phase as stated by Foct [4].

In an extension of Stoner-Mott-Friedel approach relating alloy magnetization to solute valence, Malozemoff et al. [7] explain the magnetic properties of concentrated $\mathrm{Fe}$ alloys of formulation $\mathrm{Fe}_{3} \mathrm{X}(\mathrm{X}=$ non-metal) on the basis of band-gap theory for strong ferromagnetism. Strong ferromagnetism refers here to systems where Fermi level $\left(E_{\mathrm{F}}\right)$ falls in a gap or low density of states (DOS) of either majority spins (spin up : $\uparrow$ ) or minority spins (spin down : $\downarrow$ ) bands. The deeper the gap or the lower the DOS, the stronger the ferromagnetism. Band structure calculations were done in the $\mathrm{Cu}_{3} \mathrm{Au}$-type structure for $\mathrm{Fe}_{3} \mathrm{X}$ - except for $\mathrm{Fe}_{3} \mathrm{~N}-$ independently of their real structure.

In view of the experimental and theoretical studies, it appeared relevant to us to investigate $\mathrm{Fe}_{3} \mathrm{~N}$ by calculating its electronic and magnetic properties in its real hexagonal structure and in the $\mathrm{Cu}_{3} \mathrm{Au}$-type hypothetical structure. For reasons of program capacity and structure complexity only the stoichiometric phase $3: 1$ will be considered.

\section{Crystal structures.}

2.1 HeXagonal $\mathrm{Fe}_{3} \mathrm{~N}$. - $\mathrm{Fe}_{3} \mathrm{~N}$ crystallizes in the hexagonal system with two formula units per unit cell i.e. $\mathrm{Fe}_{6} \mathrm{~N}_{2}$. In the earliest determinations of the crystal structure [1], the $\mathrm{P}_{3} 22$ space group was proposed with Fe occupying a nearly h.c.p. arrangement. In a subsequent study [2b], a less symmetrical space group i.e. P312 was adopted and used later to refine neutron diffraction spectra [5]. Within this space group which we use in our calculations, atoms are arranged as follows:

- 6 iron atoms at the 61 general positions;

- 2 nitrogen atoms at $1 \mathrm{~d}$ and $1 \mathrm{e}$ special positions and

- 4 unoccupied interstitial sites at $1 \mathrm{~b}, 1 \mathrm{f}, 1 \mathrm{a}$ and $1 \mathrm{c}$ special positions. It is at such sites that excess nitrogen enters upon approaching the higher limit of the $\varepsilon$-phase (cf. Tab. I).

Hence along the $c$ axis the structure can be considered as a succession of A-B-A-B i.e. Fe(N)-Fe-(N) layers as shown in figure 1, with $\mathrm{N}$ acting as a spacer in the Fe lattice. Vacant sites alternate with $\mathrm{N}$ in the $\mathrm{B}$ layers. They are filled in a random manner when nitrogen content increases until half of them are occupied. At such a composition figure 1 shows that 2 nitrogen atoms along $c$ would be at a distance of $c / 2$, too short to allow for the hexagonal arrangement of the atoms to be preserved. Consequently, a transformation to an orthorhombic symmetry occurs for a composition corresponding to $\mathrm{Fe}_{2} \mathrm{~N}$.

A related - but different - structure is the $\mathrm{Ni}_{3} \mathrm{Sn}$-type where $\mathrm{Fe}(\mathrm{Ni})$ and $\mathrm{N}(\mathrm{Sn})$ would be in the same layer with the sequence $\mathrm{A}-\mathrm{B}-\mathrm{A}-\mathrm{B}$ occurring as $(\mathrm{Fe}, \mathrm{N})-(\mathrm{Fe}, \mathrm{N})$, etc.

It can be noted that the hexagonal arrangement of atoms and interstices in the $\varepsilon$-phase can be constructed from an f.c.c. lattice using the cube diagonal as the hexagonal $c$-axis. This establishes a relationship between the $\gamma^{\prime}$ phase (derived from the insertion of nitrogen within f.c.c. $\mathrm{Fe}$, with $\mathrm{Fe}_{4} \mathrm{~N}$ general formula) and the $\varepsilon$-phase; at least for nitrogen deficient compositions (ex. Tab. I, row 1 and 2).

2.2 $\mathrm{Cu}_{3} \mathrm{Au}$-TYPE STRUCTURE FOR $\mathrm{Fe}_{3} \mathrm{~N}$. - In the cubic cell of ordered $\mathrm{Cu}_{3} \mathrm{Au}$ adopted for $\mathrm{Fe}_{3} \mathrm{~N}, \mathrm{Fe}(\mathrm{Cu})$ is at the face-centers $\left(\frac{1}{2}, \frac{1}{2}, 0\right.$, etc.. ) while $\mathrm{N}(\mathrm{Au})$ occupies cube corners at 0 , 
Table I. - Range of the e-iron nitride phase $\mathrm{Fe}_{3} \mathrm{~N}_{x}$.

\begin{tabular}{|c|c|c|c|c|c|c|}
\hline Row & Value of $x$ & $\%$ wt. N & $\begin{array}{c}a^{\prime}=a \sqrt{3} \\
(\text { a.u. })\end{array}$ & $c l a^{\prime}$ & $\begin{array}{c}\text { V/f.u. } \\
(\text { a.u. })^{3}\end{array}$ & [Ref.] \\
\hline 1 & 0.67 & 5.34 & 8.676 & 0.942 & 266.38 & 4 \\
\hline 2 & 0.72 & 5.69 & 8.709 & 0.943 & 269.72 & $2 \mathrm{~b}$ \\
\hline 3 & 0.74 & 5.88 & 8.732 & 0.941 & 271.28 & $2 \mathrm{~b}$ \\
\hline 4 & 1.00 & 7.71 & 8.825 & 0.934 & 278.11 & 1 \\
\hline 5 & 1.12 & 8.57 & 8.916 & 0.932 & 286.04 & $2 \mathrm{~b}$ \\
\hline 6 & 1.17 & 8.90 & 8.949 & 0.929 & 288.29 & 5 \\
\hline 7 & 1.24 & 9.38 & 8.978 & 0.928 & 290.79 & $2 \mathrm{~b}$ \\
\hline 8 & 1.27 & 9.59 & 8.997 & 0.926 & 292.01 & $2 \mathrm{~b}$ \\
\hline 9 & 1.35 & 10.14 & 9.011 & 0.926 & 293.38 & $2 \mathrm{~b}$ \\
\hline 10 & 1.48 & 10.99 & 9.049 & 0.923 & 296.14 & $2 \mathrm{~b}$ \\
\hline
\end{tabular}

$0,0$. Due to the different species involved, the simple cubic lattice ( $\mathrm{Pm} 3 \mathrm{~m})$ has an octahedral void at the cube center i.e. at $\frac{1}{2}, \frac{1}{2}, \frac{1}{2}$.

\section{Method of calculation.}

The electronic and magnetic structures of $\mathrm{Fe}_{3} \mathrm{~N}$ were calculated using the augmented spherical wave (ASW) method which is a first principles method for self consistently calculating the band structure of solids [8]. The calculations are based on the local spin density functional theory in which exchange and correlation effects are treated within the scheme of von Barth and Hedin [9] and Janak [10]. The Brillouin-zone integration was carried out for a sufficiently large number of $k$ points in the irreducible wedge in order to obtain reliable values - i.e. until no further variation occurs - of magnetic moments and total energy of the system. The matrix elements were constructed using partial waves up to $\ell_{\max }+1$ ( $_{\max }=2$ for Fe and $\ell_{\max }=1$ for $N$ ). The $\ell+1$ terms are used for the internal summations in the threecenter terms of the matrix elements. Spin-polarized calculations - i.e. for magnetic order were done in the two structures; one additional non-spin-polarized calculation was done in the $\mathrm{Cu}_{3} \mathrm{Au}$-type structure. The $\mathrm{ASW}$ method uses the atomic sphere approximation (ASA) where each atom is surrounded by a sphere within which the potential and the charge density are assumed to be spherically symmetric. The space outside the spheres is not accounted for but the sum of the volumes of all the spheres is equal to the volume of the unit cell.

In hexagonal $\mathrm{Fe}_{3} \mathrm{~N}$ as well as in $\mathrm{Cu}_{3} \mathrm{Au}$-type structure the presence of unoccupied sites led us to introduce empty spheres (ES) i.e. pseudo-atoms with $Z=0$ within the ASA. These empty spheres prevent a too important overlap of the atomic spheres and allow for a smooth 


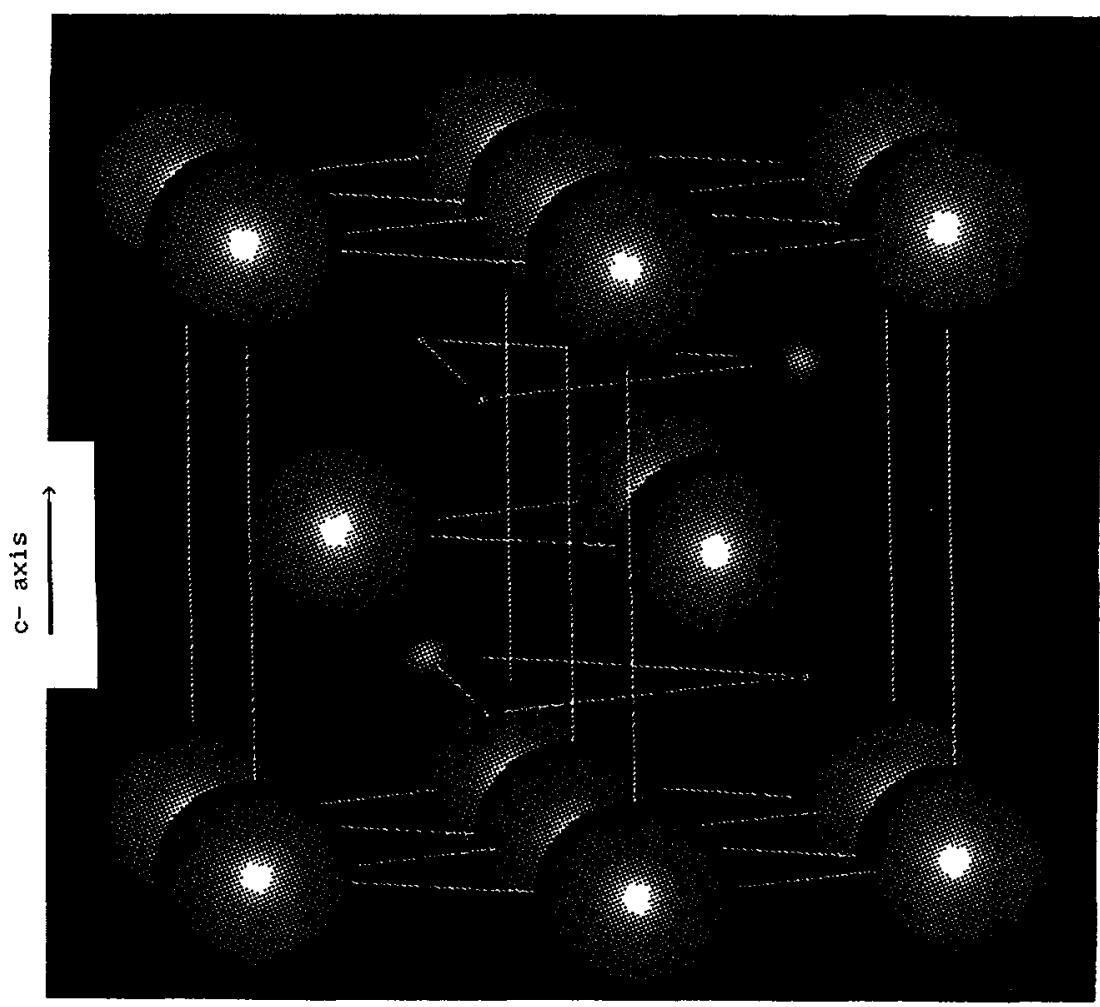

Fig. 1. - Hexagonal structure of $\mathrm{Fe}_{3} \mathrm{~N}$ showing the succession of $\mathrm{Fe}-\mathrm{N}-\mathrm{Fe}-\mathrm{N}$ layers along $c$-direction. With decreasing size, the spheres correspond to $\mathrm{Fe}, \mathrm{N}$ and vacancies respectively. Empty spheres are introduced at vacant sites.

variation of the potential in the interatomic region. They also contain the "tails " of the spherical wave functions and would account for possible covalency effects [11].

\section{ASW calculations of hexagonal $\mathrm{Fe}_{3} \mathrm{~N}$.}

Using the lattice parameters firstly determined for stoichiometric $\mathrm{Fe}_{3} \mathrm{~N}$ by $\mathrm{X}$-ray diffraction (Tab. I, row 4) and those determined by neutron diffraction for a nitrogen-excess nitride (Tab. I, row 6) we have achieved two sets of spin-polarized calculations. The Brillouin-zone integration was carried out for 80 independent $k$-points on a uniform mesh in the irreducible wedge. The self consistency cycle was run until energy convergence better than 1 mRyd was achieved (1 Ryd = $13.6 \mathrm{eV})$.

The obtained partial charges and magnetic moments are given in table IIa and $b$ respectively.

4.1 ELECTRON DISTRIBUTION. - In both sets of calculations, there is hardly any charge transfer between nitrogen and iron. Nitrogen however loses electrons to neighboring ES. Excess charge on iron arises likely from a hybridization of its states with those of $\mathrm{N}$ and ES as it will be shown in the discussion of the densities of states (DOS). From table IIa and $b$ the $s$ and $\mathrm{p}$ partial charges of $\mathrm{Fe}$ do not change with volume and their sum for majority spins is equal to 0.64 and 0.65 respectively. Hence volume effects seem to affect the $3 \mathrm{~d}$ band solely 
Table II. - Local partial charges for spin-up $(\uparrow)$ and spin-down $(\downarrow)$ directions for $\mathrm{Fe}_{3} \mathrm{~N}$.

a) Lattice parameters from reference [1] : $a=8.82565$ a.u. ; cla $=0.93429 ; V=556.225$ (a.u. $)^{3}, r(\mathrm{Fe})=2.61916$ a.u. $; r(\mathrm{~N})=r(\mathrm{ES})=1.60882$ a.u.

\begin{tabular}{|l|c|c|c|c|c|c|c|c|c|c|}
\hline & $n_{\mathrm{s} \uparrow}$ & $n_{\mathrm{s} \downarrow}$ & $n_{\mathrm{p} \uparrow}$ & $n_{\mathrm{p} \downarrow}$ & $n_{\mathrm{d} \uparrow}$ & $n_{\mathrm{d} \downarrow}$ & $n_{\mathrm{f} \uparrow}$ & $n_{\mathrm{f} \downarrow}$ & $M_{\mu_{\mathrm{B}}}$ & $Q_{\mathrm{tot}}$ \\
\hline $\mathrm{Fe}$ & 0.26 & 0.26 & 0.38 & 0.39 & 4.29 & 2.32 & 0.06 & 0.06 & 1.95 & 8.05 \\
\hline $\mathrm{N}$ & 0.60 & 0.60 & 1.38 & 1.44 & 0.02 & 0.01 & - & - & -0.05 & 4.07 \\
\hline $\mathrm{ES}$ & 0.12 & 0.14 & 0.06 & 0.06 & - & - & - & - & -0.01 & 0.38 \\
\hline
\end{tabular}

$$
E_{\mathrm{F}}=0.820 \mathrm{Ryd} ; \quad E_{\mathrm{var}}=-15463.227104 \mathrm{Ryd} \quad \mu_{\mathrm{av} .}=1.44 \mu_{\mathrm{B}} .
$$

b) Lattice parameters from reference [5] : $a=8.94896$ a.u. ; cla $=0.92860 ; V=576.337$ (a.u. $)^{3}, r(\mathrm{Fe})=2.65035$ a.u. $; r(\mathrm{~N})=r(\mathrm{ES})=1.62798$ a.u.

\begin{tabular}{|l|l|c|c|c|c|c|c|c|c|c|}
\hline & $n_{\mathrm{s} \uparrow}$ & $n_{\mathrm{s} \downarrow}$ & $n_{\mathrm{p} \uparrow}$ & $n_{\mathrm{p} \downarrow}$ & $n_{\mathrm{d} \uparrow}$ & $n_{\mathrm{d} \downarrow}$ & $n_{\mathrm{f} \uparrow}$ & $n_{\mathrm{f} \downarrow}$ & $M_{\mu_{\mathrm{B}}}$ & $Q_{\text {tot }}$ \\
\hline $\mathrm{Fe}$ & 0.26 & 0.27 & 0.38 & 0.39 & 4.35 & 2.26 & 0.06 & 0.06 & 2.07 & 8.04 \\
\hline $\mathrm{N}$ & 0.61 & 0.61 & 1.39 & 1.45 & 0.02 & 0.01 & - & - & -0.05 & 4.10 \\
\hline $\mathrm{ES}$ & 0.12 & 0.14 & 0.06 & 0.06 & - & - & - & - & -0.01 & 0.38 \\
\hline
\end{tabular}

$$
E_{\mathrm{F}}=0.784 \mathrm{Ryd} ; \quad E_{\mathrm{var}}=-15463.298554 \mathrm{Ryd} \quad \mu_{\mathrm{av} .}=1.54 \mu_{\mathrm{B}} .
$$

leading to the development of the magnetic moment and to its change with volume. There is a slight unbalance in polarization of the $2 p$ states of nitrogen between majority $(\uparrow)$ and minority $(\downarrow)$ spins in favor of the latters whence the negative value of the moment.

4.2 MAGNETIC MOMENTS. - As it is known, the magnetic moment arises from an unbalance of charge distribution between $\uparrow$ and $\downarrow$ spins. From table II it is mainly the 3d intra-band polarization which provides the moment for $\mathrm{Fe} . \mathrm{N}$ and $\mathrm{ES}$ hardly bear any moment. The calculated magnetic moments of Fe i.e. 1.95 (Tab. IIa) and $2.07 \mu_{B}$ (Tab. IIb) are close to the value recently computed in a work we came across during the preparation of this paper, i.e. $1.94 \mu_{B}$ [12]. The value of the magnetization averaged over the unit cell are 1.44 and $1.54 \mu_{B}$. The difference between our two calculated moments is due to the lattice expansion for the latter calculation, in agreement with [12]. This feature was addressed by one of us in recent calculations of iron rich nitride $\mathrm{Fe}_{8} \mathrm{~N}$ [13]. Both values of the average magnetization are higher than the experimental one of $1.33 \mu_{B}$ [5]. Although the latter was measured for an expanded lattice, it needs to be stressed that experimentally the lattice expansion is induced by excess nitrogen whereas our calculations merely simulate stoichiometric $\mathrm{Fe}_{3} \mathrm{~N}$ at experimental lattice constant as well as in an expanded lattice. On another hand for the most $\mathrm{N}$-deficient $\varepsilon$-phase having the lowest cell volume i.e. $\mathrm{Fe}_{3} \mathrm{~N}_{0.67}$ (Tab. I, row 1), magnetic 
measurements yielded a value of $2.3 \mu_{\mathrm{B}}$ for $\mathrm{Fe}$ moment. Consequently two phenomena with opposite effects occur :

i) a slight lattice expansion of an Fe lattice should lead to a moment increase [13] ; but simultaneously,

ii) increasing amount of nitrogen leads to a decrease of the moment borne by $\mathrm{Fe}$ [4].

The decrease of the magnetic moment of $\mathrm{Fe}$ in the nitride is likely to occur throughout a mechanism of spin pairing between $\mathrm{N}(2 \mathrm{p})$ states and $\mathrm{Fe}(3 \mathrm{~d})$ ones in a similar way as within the cubic nitride $\gamma^{\prime}-\mathrm{Fe}_{4} \mathrm{~N}$ [14, and therein cited references]. This point will be further addressed in the analysis of the DOS.

Relationship with other magnetic systems. - The nearly constant value of $\operatorname{sp}(\uparrow)$ in the two sets of calculations i.e. $n_{s_{\uparrow}}+n_{p_{\uparrow}}=0.64$ and 0.65 respectively and its proximity to $0.6:$ a value characteristic of several alloy systems led us to envisage a possible relationship between the average magnetic moment and the electron count for $\mathrm{Fe}_{3} \mathrm{~N}$ throughout the Slater-PaulingFriedel (SPF) curve [15] plotted for ferromagnets. Such a curve is the plot of $\mu_{\text {av. }}=Z_{\mathrm{m}}+0.6$ where $\mu_{\text {av. }}$ is the average magnetization per atom in the unit cell and $Z_{\mathrm{m}}$ is the magnetic valence defined as : $Z_{\mathrm{m}}=2 N_{\mathrm{d} \uparrow}-Z_{\mathrm{val}}\left(N_{\mathrm{d} \uparrow}\right.$ : number of d electrons for majority spins i.e. 5 for elements with filled majority-spin half-band $-\mathrm{Fe}, \mathrm{Co}$ and $\mathrm{Ni}-$ and 0 for $\mathrm{N}$; $Z_{\text {val. }}$. number of valence electrons i.e. 8 for $\mathrm{Fe}$ and 3 for $\mathrm{N}$ ). Hence $Z_{\mathrm{m}}(\mathrm{Fe})=2$, $Z_{\mathrm{m}}(\mathrm{N})=-3$ and $Z_{\mathrm{m}}\left(\mathrm{Fe}_{3} \mathrm{~N}\right) / \mathrm{at} .=0.75$ can be computed. Taking the average magnetization per atom from table Ila i.e. $1.44 \mu_{B}$ and the experimental average magnetization of $1.33 \mu_{B}$ the $\left(Z_{\mathrm{m}}, \mu_{\text {ay. }}\right)$ points corresponding to $\mathrm{Fe}_{3} \mathrm{~N}$ lie between $\mathrm{Co}$ and $\mathrm{Ni}$ - strong ferromagnets together with other alloy systems on the right hand side of the SPF as given in reference [15, Fig. 62]. Consequently $\mathrm{Fe}_{3} \mathrm{~N}$ can be assigned a strong ferromagnet behavior within this framework.

4.3 FIXED SPIN MOMENT CALCULATIONS. - In order to check that the calculated magnetic moment actually corresponds to a minimum of total energy, fixed spin moment calculations were undertaken. Such calculations constrain total magnetization to a fixed value. For successive values of the magnetization, calculations are self-consistently converged yielding the total energy for each one of them [16]. Such calculations (done with the lattice parameters given in Tab. I, row 6 and used for Tab. IIb), yielded one energy minimum for a total magnetization of $12.30 \mu_{B}$; i.e. per two formula units of $\mathrm{Fe}_{3} \mathrm{~N}$ and a resulting moment of $2.07 \mu_{\mathrm{B}}$ per $\mathrm{Fe}$ atom. This is in agreement with the value of the moment of $\mathrm{Fe}$ in table $\mathrm{Hb}$. Hence the variation of energy with magnetization points to the existence of one magnetic state for $\mathrm{Fe}_{3} \mathrm{~N}$ whereas h.c.p.-Fe at a sphere radius of 2.66 a.u. (atomic units, 1 a.u. $=0.529 \AA$ ) i.e. close to the value of the $\mathrm{Fe}$ sphere radius in our calculations (cf. $\mathrm{Tab}$. IIb), was shown to exhibit a non-magnetic ground state at low energy and a magnetic state at a higher energy [17, Fig. 2].

4.4 INTERNAL FIELDS. - The contribution of the Fermi contact terms to the internal fields $H_{\mathrm{i}}$ are calculated from the unbalance of spin-densities of the s-electrons at the nucleus following the relationship :

$$
H_{\mathrm{FC}}=-\frac{8}{3} \pi \gamma_{\mathrm{N}}\left\{\phi_{\uparrow}(0)^{2}-\phi_{\downarrow}(0)^{2}\right\}
$$

where $\gamma_{N}$ is the nuclear gyromagnetic ratio and $\phi_{\uparrow}(0)^{2}$ and $\phi_{\downarrow}(0)^{2}$ the charge densities of the $\mathrm{s}$-electrons at the nucleus for the two spin directions. The Fermi-contact terms at Fe site are -200 and $-212 \mathrm{kOe}$ respectively for the calculations given in tables IIa and IIb. A measured value for a sample close to $3: 1$ composition of - 244 kOe [19] shows a relative agreement. 


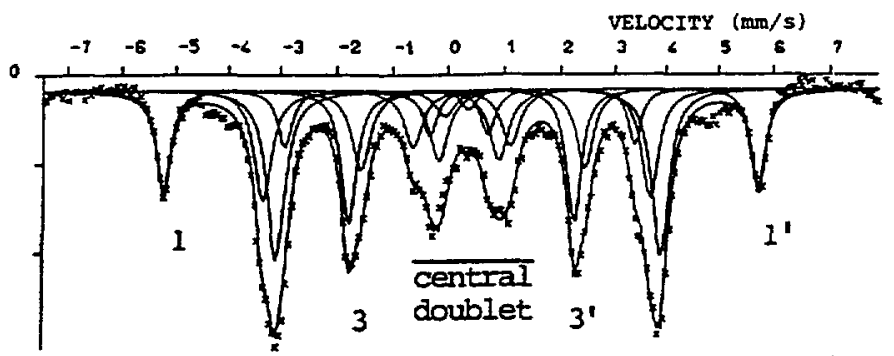

a)

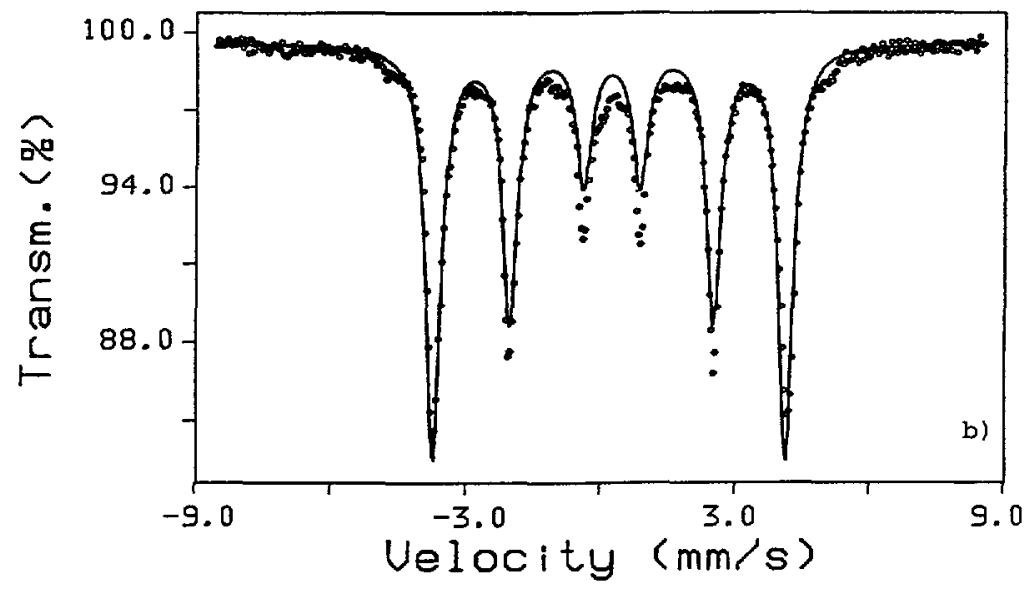

Fig. 2. - Mössbauer absorption spectra of $y^{\prime}-\mathrm{Fe}_{4} \mathrm{~N}$ (a) and $\varepsilon-\mathrm{Fe}_{3} \mathrm{~N}$ (b).

Mössbauer measurements of the $\varepsilon$-phase at a nitrogen deficient composition called $\varepsilon-\mathrm{Fe}_{4} \mathrm{~N}$ [4] allowed to determine for the two crystallographic sites two different internal fields: $H_{\mathrm{I}}=-298.1 \mathrm{kOe}$ and $H_{\mathrm{II}}=-221.8 \mathrm{kOe}$. These two values are close to those found for $\gamma^{\prime}-\mathrm{Fe}_{4} \mathrm{~N}$ for similarly labeled sites [18] and can be understood in view of the structural relationship mentioned in paragraph 2.1. Figure 2 shows the Mössbauer spectra of $\gamma^{\prime}-\mathrm{Fe}_{4} \mathrm{~N}$ (a) and $\varepsilon-\mathrm{Fe}_{3} \mathrm{~N}$ (b, [19]). The spectrum of the former was deconvoluted [5] into two sets of lines called sextets : « $1 \& 1^{\prime} »$ and $« 3 \& 3^{\prime} »$ attributed to the two Fe sites I and II respectively and one central doublet assigned to very fine superparamagnetic particles present into the powder sample. The spectrum of $\mathrm{Fe}_{3} \mathrm{~N}(2 \mathrm{~b})$ shows comparatively only a sextet of type II as well as a central doublet whereas the type « $1 »$ sextets are absent [4]. The presence of one Fe sublattice in $\mathrm{Fe}_{3} \mathrm{~N}$ is hence confirmed. It needs to be said that the present analysis of the Mössbauer spectra is only qualitative since, for instance, no electric field gradient effects are accounted for ; those will be extensively addressed elsewhere [19].

4.5 DENSITIES OF STATES (DOS). - The site- and spin-projected DOS are shown in figure $3 a(F e), b(N)$ and $c(E S)$. They are representative of the two sets of calculations of table II which yield in similar DOS. Energies are counted with respect to $E_{\mathrm{F}}$ at $\cong 11 \mathrm{eV}$.

From left to right the DOS present the following features :

* 2s states below $-13 \mathrm{eV}$;

* $2 \mathrm{p}$ states in the range $-8,-5 \mathrm{eV}$ and

* $3 \mathrm{~d}$ states in the range $-5,0 \mathrm{eV}$ i.e. crossed by $E_{\mathrm{F}}$.

The presence of a finite DOS at $E_{\mathrm{F}}$ is a general feature of iron nitrides and is in agreement 


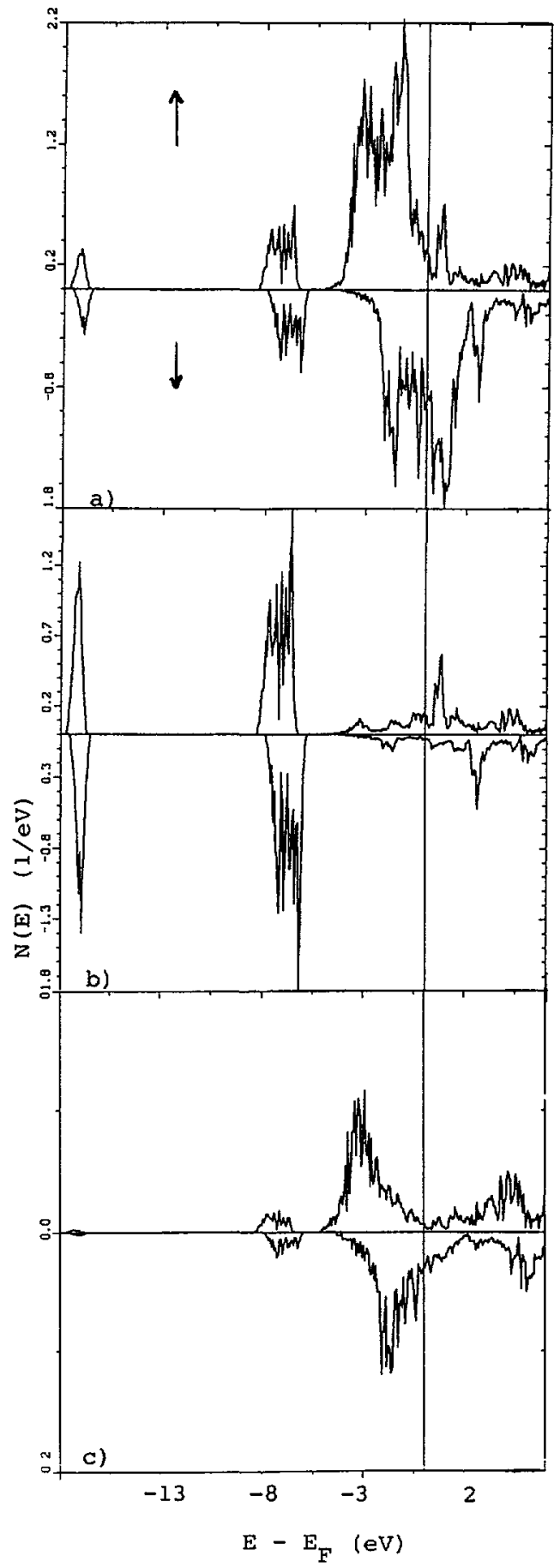

Fig. 3. - Densities of states of $\mathrm{Fe}_{3} \mathrm{~N}$ projected along the two spin directions over the 3 different sites of the hexagonal $\mathrm{Fe}_{3} \mathrm{~N}$ lattice : a) $\left.\mathrm{Fe}, \mathrm{b}\right) \mathrm{N}$ and c) ES. Energies are counted with respect to $E_{\mathrm{F}}$. 
with their metallic type behavior $[13,14]$ so that they can be considered as itinerant ferromagnets.

In the following we shall analyse the DOS at each lattice site and suggest correlations between them.

The Fe DOS (Fig. 3a) are mainly dominated by the 3d states. The shift between majority spins and minority spins i.e. intra-band polarization, provides the magnetic moment. They present similarities with fcc-type d-DOS as previously observed for $\gamma^{\prime}-\mathrm{Fe}_{4} \mathrm{~N}$ for iron at face centers [14]. The majority spin states constitute a nearly half-filled d-band as they lie below $E_{\mathrm{F}}$ with a low DOS at $E_{\mathrm{F}}$. This feature suggests a strong ferromagnet behavior in accordance with the assessments of paragraph 4.2.1. There is a strong mixing between the states of nitrogen and those of $\mathrm{Fe}$ due to similarities between $2 \mathrm{p}$ and $3 \mathrm{~d}$ DOS - as what one would expect for a covalently bonded compound. This interaction gives rise to $\sigma$ and $\pi$ bonds (notice the well separated two-fold families of peaks between -7 and $-8 \mathrm{eV}$ ) with their antibonding counterparts above (isolated two-fold peak) $E_{\mathrm{F}}$ and at $E_{\mathrm{F}}$ (notice the three-fold peak for $\uparrow$ spin DOS) respectively. The presence of the latter peak structure due to nitrogen allowed to suggest a weak itinerant ferromagnet behavior for $\mathrm{NiFe}_{3} \mathrm{~N}$ [20].

The $2 \mathrm{~s}$ and $2 \mathrm{p}$ states of nitrogen (Fig. $3 \mathrm{~b}$ ) show a certain amount of structure due to a nonregular environment made of $\mathrm{Fe}$ and $\mathrm{ES}$. The formers can be considered as semi-core states and they play a small role in the bonding whereas $2 p$ states play a role in the bonding with $\mathrm{Fe}$ as stated above.

A $\sigma$ bond occurs between an iron orbital and one N-2p half filled orbital. The formation of such a bond reduces the number of d-electrons that can develop a magnetic moment at Fe by spin pairing. This situation is similar to $\gamma^{\prime}-\mathrm{Fe}_{4} \mathrm{~N}$ where the magnetic moments differ by $1 \mu_{B}$ between the two Fe sublattices due to one preferential $\mathrm{Fe} . . . \mathrm{N}$ bond [14].

The shape of the ES-DOS (Fig. 3c) gives a major contribution to a mixing with the d-Fe states hence transferring charge into them (Tab. II).

The general shape of the DOS of hexagonal $\mathrm{Fe}_{3} \mathrm{~N}$ is different from those of the $\mathrm{Fe}_{3} \mathrm{X}$ compounds calculated by Malozemoff et al. [7], especially at $E_{\mathrm{F}}$ where no gap could be observed upon plotting the $\ell$-projected DOS for $F$ in either spin direction. This point will be further discussed in next section.

\section{ASW calculations of $\mathrm{Fe}_{3} \mathrm{~N}$ in the $\mathrm{Cu}_{3} \mathrm{Au}$ structure.}

In order to enable for comparisons with the $\mathrm{Fe}_{3} \mathrm{X}$ homologues [7], two sets of calculations were achieved : spin-polarized and non spin-polarized calculations. The lattice constant of the cubic unit cell was derived from the volume of the hexagonal cell of stoichiometric $\mathrm{Fe}_{3} \mathrm{~N}$ (Tab. I, row 4) i.e. 6.52741 a.u. The two sets of calculations were done using equal sphere radii for the respective species in the two calculations. The Brillouin-zone integration was carried out for 35 independent $k$-points. The results of the calculations are given in table III.

From table IIIa it can be seen that the intra-band polarization of the $3 \mathrm{~d}$ states of Fe is higher - i.e. more filling of the majority-spin band - than in the hexagonal structure with a net result of a higher moment. Hence the tendency to a strong ferromagnet behavior could be suggested. There is very little charge transfer between $\mathrm{Fe}$ and $\mathrm{N}$, lower than in the hexagonal structure (Tabs. IIa and $b$ ). The sum of $s$ and $p$ partial charges of $F e(\uparrow)$ i.e. 0.65 does not change with respect to their total value in the former calculation in the hexagonal structure. This seems to be a structure independent feature due to $\mathrm{Fe} \ldots \mathrm{N}$ interaction. The volume effect induced by the increase of the sphere radius of $\mathrm{Fe}$ in the cubic cell compared to its value in the hexagonal one solely affects the $d$ states. The average magnetization per atom is equal to $2.25 \mu_{B}$ i.e. much larger than either the experimental or formerly calculated values, moreover it does not fit on the SPF curve as above. For $\mathrm{Fe}_{3} \mathrm{X}(\mathrm{X}=\mathrm{B}, \mathrm{Si}$ and $\mathrm{Sn})$ calculated in 
Table III. - ASW results for $\mathrm{Cu}_{3} \mathrm{Au}-t y p e \mathrm{Fe}_{3} \mathrm{~N}(a=6.52741$ a.u.). a) Local partial charges for spin-up (†) and spin-down $(\downarrow)$ directions.

\begin{tabular}{|l|c|c|c|c|c|c|c|c|c|c|}
\hline & $n_{\mathrm{s} \uparrow}$ & $n_{\mathrm{s} \downarrow}$ & $n_{\mathrm{p} \uparrow}$ & $n_{\mathrm{p} \downarrow}$ & $n_{\mathrm{d} \uparrow}$ & $n_{\mathrm{d} \downarrow}$ & $n_{\mathrm{f} \uparrow}$ & $n_{\mathrm{f} \downarrow}$ & $M_{\mu_{\mathrm{B}}}$ & $Q_{\text {tot }}$ \\
\hline $\mathrm{Fe}$ & 0.31 & 0.30 & 0.34 & 0.32 & 4.68 & 1.94 & 0.06 & 0.04 & 2.79 & 7.98 \\
\hline $\mathrm{N}$ & 0.87 & 0.82 & 1.48 & 1.34 & 0.005 & 0.004 & - & - & 0.18 & 4.53 \\
\hline $\mathrm{ES}$ & 0.16 & 0.17 & 0.12 & 0.07 & - & - & - & - & 0.03 & 0.53 \\
\hline
\end{tabular}

$$
E_{\mathrm{F}}=0.827 \mathrm{Ryd} ; \quad E_{\mathrm{var}}=-7731.18488 \mathrm{Ryd}
$$

b) Local partial charges for total spin.

\begin{tabular}{|c|c|c|c|c|c|}
\hline & $n_{\mathrm{s}}$ & $n_{\mathrm{p}}$ & $n_{\mathrm{d}}$ & $n_{\mathrm{f}}$ & $Q_{\text {tot }}$ \\
\hline $\mathrm{Fe}$ & 0.59 & 0.64 & 6.67 & 0.09 & 8.0 \\
\hline $\mathrm{N}$ & 1.75 & 2.70 & 0.009 & - & 4.46 \\
\hline $\mathrm{ES}$ & 0.33 & 0.21 & - & - & 0.54 \\
\hline
\end{tabular}

$$
E_{\mathrm{F}}=0.798 \mathrm{Ryd} ; \quad E_{\mathrm{var}}=-7731.10653 \mathrm{Ryd} .
$$

the same crystal structure the magnitude of the moment of the non metal varies from -0.23 for $\mathrm{X}=\mathrm{B}$ to -0.10 for $\mathrm{X}=\mathrm{Sn}[7]$. This is markedly opposed to the behavior of $\mathrm{N}$ whose large magnetic moment has a positive sign.

The site and spin-projected DOS are given in figure 4 for the spin-polarized calculation, moreover the $\mathfrak{l}$-projected DOS at Fe are shown as well. The general shape of the Fe-DOS is very similar to the $\mathrm{DOS}$ of $\mathrm{Fe}_{3} \mathrm{~B}$ [7]. The difference in energy between the $\mathrm{N}-2 \mathrm{p}$ and $\mathrm{Fe}-3 \mathrm{~d}$ states is not sufficient to cause their separation contrary to what it can observed between the same partial DOS in figure 3. Comparing figures 3 and 4 the $\mathrm{N}-2 \mathrm{p}$ and $2 \mathrm{~s}$ DOS lie lower in energy in figure 3. This could point to a different ionic behavior whereby $\mathrm{Fe}_{3} \mathrm{~N}$ would be «more ionic » in its real structure (see the larger charge transfer between $F e$ and $N$ in Tab. II). On another hand the mixing between $\mathrm{Fe}$ and $\mathrm{N}$ states in the $\mathrm{Cu}_{3} \mathrm{Au}$ structure leads to a large polarization of the $N$ states with a large magnetic moment of $N\left(0.18 \mu_{B}\right.$. Tabl. III) contrary to the lower moment of $N$ in the hexagonal structure $\left(-0.05 \mu_{B}\right.$. Tab. II).

Observation of the $\ell$-projected (Fig. $4 b$ ) $: \mathcal{l}=0$ and (Fig. 4c) $: \mathcal{l}=1$ ) DOS for Fe clearly shows a gap at $E_{\mathrm{F}}$ for the majority spins. The feature of the minimum DOS at $E_{\mathrm{F}}$ for $\mathrm{s}(\mathrm{Fe})$ and $\mathrm{p}(\mathrm{Fe})$ states was seen as well for paramagnetic calculations of $\mathrm{Fe}_{3} \mathrm{~N}$. The important requirement of the «band gap theory » of strong ferromagnetism is that hybridization of the $\mathrm{sp}$ states with the $\mathrm{d}$ states results in a depression near the Fermi level of the density of $\mathrm{sp}$ states, that is, states that might respond to the stronger metalloid sp potential \{sic [7]\}. This condition seems to be met for $\mathrm{Fe}_{3} \mathrm{~N}$ calculated in this structure.

Considering the bonding character of $\mathrm{Fe} . . \mathrm{N}$, the $\mathrm{N}-2 \mathrm{p}$ DOS can be assigned $\sigma$ and $\pi$ 


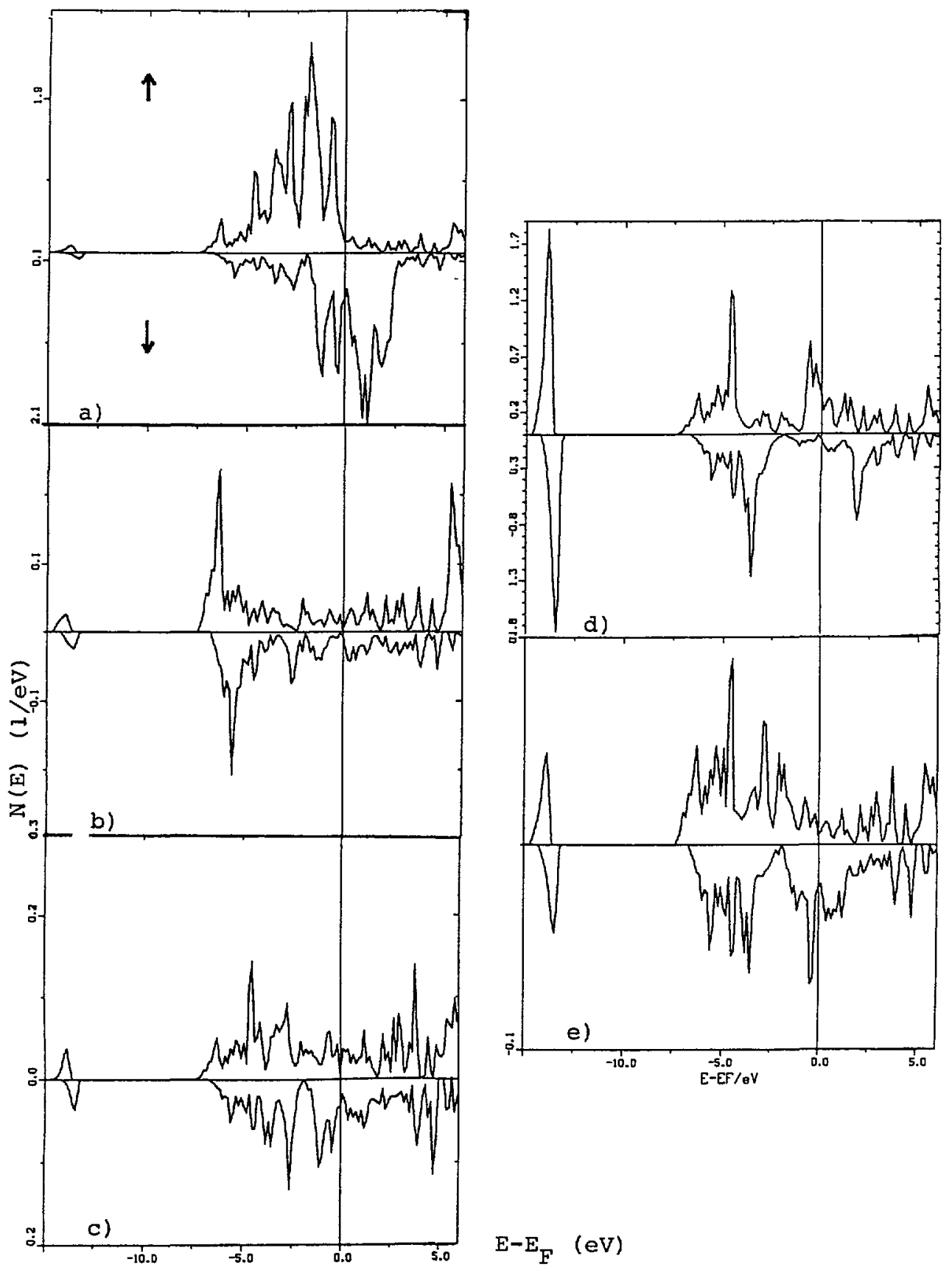

Fig. 4. - Densities of states (DOS) of $\mathrm{Fe}_{3} \mathrm{~N}$ projected along the two spin directions and along over the 3 different sites of $\mathrm{Cu}_{3} \mathrm{Au}$-type $\mathrm{Fe}_{3} \mathrm{~N}$ lattice : a) $\mathrm{Fe}$, d) $\mathrm{N}$ and e) $\mathrm{ES} . \ell$-projected DOS for $\mathrm{Fe}$ are shown in b) $\ell=0$ and c) $\ell=1$. Energies are counted with respect to $E_{\mathrm{F}}$. 
bonding and antibonding behavior in the same way as in the hexagonal phase. Within this framework, a noticeable feature is observed in figure $4 \mathrm{~d}$ where antibonding states $(\uparrow)$ arising from $\mathrm{N}$ sit on $E_{\mathrm{F}}$ (mostly $\pi^{*}$, notice the three-fold peak structure in relation with Fig. $3 \mathrm{~b}$ ). It needs to be stressed that the position of this peak did not change with other calculations done at larger lattice constants. A major role seems to be played by nitrogen by introducing DOS at $E_{\mathrm{F}}$ as in the hexagonal structure.

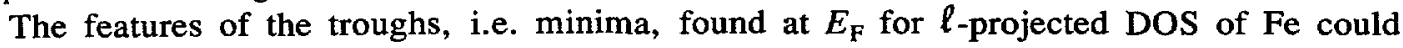
assimilate $\mathrm{Fe}_{3} \mathrm{~N}$ to its $\mathrm{Fe}_{3} \mathrm{X}$ homologues. However further calculations of partial DOS of X the $\mathrm{Fe}_{3} \mathrm{X}$ would be needed to check whether $\mathrm{X}$ introduces DOS at $E_{\mathrm{F}}$ in a similar way as $\mathrm{N}$.

Considering the total variational energies in table IIIa and $\mathrm{b}$, hypothetical $\mathrm{Cu}_{3} \mathrm{Au}$-type $\mathrm{Fe}_{3} \mathrm{~N}$ would be stabilized in a spin-polarized state. However (cf. Tabs. II and III) there is a net energy stabilization for the hexagonal form by $-0.4287 \mathrm{eV}$ per formula unit with respect to $\mathrm{Cu}_{3} \mathrm{Au}$-type one. Hence it is not likely to stabilize $\mathrm{Fe}_{3} \mathrm{~N}$ in such a cubic structure.

\section{Conclusions.}

In this work the electronic and magnetic properties of $\mathrm{Fe}_{3} \mathrm{~N}$ were studied by the ASW method in two crystal structures, the hexagonal experimental one and a hypothetical cubic one.

Whereas lattice expansion should lead to a moment increase when $\mathrm{N}$ is introduced in h.c.p.$\mathrm{Fe}$, this effect is hindered by the spin pairing between nitrogen and iron states leading to a reduced moment. That allows to asses the differences observed between experimental results and our calculations for the magnetic moment.

In connection with the relationship between average magnetization and electron count, $\mathrm{Fe}_{3} \mathrm{~N}$ was assigned a strong ferromagnet behavior.

The calculations have shown that independtly of the structure :

- the N-Fe interaction keeps the $s$ and $p$ electron count of $\mathrm{Fe}$ constant for the majority states ; and,

- nitrogen is likely to play a role in the magnetic behavior of the nitride by introducing states at $E_{\mathrm{F}}$.

\section{Acknowledgments.}

One of us S.M. thanks the Alexander von Humboldt foundation for a grant in the Fed. Rep. of Germany. Fruitful discussions with Dr. J. Sticht of the Technical University of Darmstadt (Germany) are acknowledged.

\section{References}

[1] Hendricks B. and Kosting R., Z. Krystallogr. 74 (1930) 511.

[2] a) JACK K. H., Proc. Roy. Soc. A 208 (1951) 200-216;

b) JACK K. H., Acta Cryst. 5 (1951) 401.

[3] Bridelle J., Ann. Chim. 10 (1955) 824.

[4] Foct J., Thèse de Doctorat d'Etat, Université Nancy 1, France (1973).

[5] Robins M. and White J. G., J. Phys. Chem. Solids 25 (1964) 717.

[6] EICKel K. H. and PITSCH W., Phys. Status Solidis 39 (1970) 121.

[7] Malozemoff A. P., Williams A. R. and Moruzzi V. L., Phys. Rev. B 29 (1984) 1620.

[8] Williams A. R., Kübler J. and Gelatt Jr. C. D., Phys. Rev. B 19 (1979) 6094.

[9] von Barth U. and Hedin L., J. Phys. Colloq. France 33 (1972) C 5-1629.

[10] JANAK J. F., Solid State Commun. 25 (1978) 53.

[11] Rompa H. W., Schuurmans M. F. and Williams F., Phys. Rev. Lett. 52 (1984) 675. 
[12] Sakuma A., J. Magn. Magn. Mat. 102 (1991) 127.

[13] Matar S., Z. Physik B, Cond. Matt. 87 (1992) 91.

[14] Matar S., Mohn P., Demazeau G. and Siberchicot B., J. Phys. France 49 (1988) 1761.

[15] KÜBLER J. and EYERT V., «Electronic structure calculations 》 in Materials Science and Technology, Haasen, Kramer Eds., Electronic and Magnetic properties of Metals and Ceramics, Buschow Ed., VCH, Verlaggesellschaft, Weinheim, Germany.

[16] Moruzzi V. L., Marcus P. M., Schwarz K. and Mohn P., Phys. Rev. B 34 (1986) 1784.

[17] KÜBleR J., Solid State Commun. 72 (1989) 631.

[18] Andriamandroso D., Fefilatiev L., Demazeau G., Fournes L. and Pouchard M., Mat. Res. Bull. 19 (1984) 1187.

[19] Pösinger A., Steiner W. and Matar S., unpubl. (1992).

[20] Mohn P., Schwarz K., Matar S. and Demazeau G., Phys. Rev. B 45 (1992) 4000. 